\title{
Can a Traditional Chinese Medicine Contribute to a Cure for HIV?
}

Thomas J. Hope

$\mathbf{T}$ HE LeTter TO THE EDITOR by Wang, entitled "Longterm survival of AIDS patients treated with only traditional Chinese medicine," describes a preliminary case series started by a small Chinese company in 2001 that examined the impact of a traditional Chinese medicine (TCM) on nine individuals with apparent symptoms of AIDS. The HIVpositive status of the nine individuals was confirmed in 2002. The company went bankrupt in 2009 , availability of the TCM ended, and most of the records were lost. However, the nine participants continued in good health and remain in that state to this date. There were no placebo controls. A highly sensitive polymerase chain reaction assay done in 2016 found that eight of the nine had undetectable viral loads, while the ninth was barely detectable. Limited documentation shows that all the individuals had much higher viral loads in the past. There are no records from before the start of TCM use, which was initiated based on AIDS-like symptoms.

There are clearly limitations to interpreting the outcome of the nine HIV-infected individuals described in this case series. The lack of viral quantification before the initiation of TCM, an incomplete understanding of the TCM components, small group size, and the lack of placebo controls make the observations presented significantly below the scientific standard normally required for publication in AIDS Research and Human Retroviruses. However, I believe that the observations presented are interesting enough that they should be released to the public with a complete description of the caveats and shortcomings. I believe that there should be some effort to further explore this phenomenon. For example, the viral reservoirs could be defined in the nine individuals if they are available. It is known that a large population of cells containing defective HIV is established early in the natural history of infection. ${ }^{1}$ The echoes of this viral shrapnel should be readily detectable in the nine individuals and have a sequence diversity consistent with years of viral selection by immune responses. ${ }^{2}$ Furthermore, the potential antiviral ability of the TCM can be evaluated in tissue culture and animal models to determine feasibility, potency, and mech- anism of action. If these studies are consistent with an impact of the TCM on HIV, a properly powered placebo controlled study could be done.

The recent report that a pharmaceutical antibody (vedolizumab) used for treatment of inflammatory bowel disease could lead to viral control in rhesus macaque models reinforces the possibility that impactful treatments can come from unexpected places. ${ }^{3}$ The publication of this Letter to the Editor provides a documentation of the limited results in hand and puts these observations into the hands of the HIV research community. It should be possible to quickly determine if this story about a TCM represents a breakthrough or a statistical anomaly. Good scientific follow-up will provide an answer.

\section{References}

1. Bruner KM, Murray AJ, Pollack RA, et al:: Defective proviruses rapidly accumulate during acute HIV-1 infection. Nat Med 2016;22:1043-1049.

2. Palma $\mathrm{P}$, Zangari $\mathrm{P}$, Alteri $\mathrm{C}$, et al.: Early antiretroviral treatment (eART) limits viral diversity over time in a longterm HIV viral suppressed perinatally infected child. BMC Infect Dis 2016;16:742.

3. Byrareddy SN, Arthos J, Cicala C, et al.: Sustained virologic control in SIV+ macaques after antiretroviral and alpha4beta7 antibody therapy. Science 2016;354:197-202.

Address correspondence to: Thomas J. Hope, PhD Department of Cell and Molecular Biology Feinberg School of Medicine Northwestern University 9-290, Lurie Building 303 East Superior Chicago, IL 60611

E-mail: thope@northwestern.edu

Department of Cell and Molecular Biology, Feinberg School of Medicine, Northwestern University, Chicago, Illinois. 